\title{
Ion-assisted deposition of silicon nitride films using electron cyclotron resonance plasma
}

\author{
K. Deenamma Vargheese and G. Mohan Rao ${ }^{\mathrm{a})}$ \\ Thin Films Laboratory, Department of Instrumentation, Indian Institute of Science, Bangalore 560012, \\ India
}

\begin{abstract}
Ion-assisted deposition (IAD) is one of the most widely used techniques for the deposition of thin films. Electron cyclotron resonance (ECR) ion sources are ideal for ion-assisted deposition due to high ionization efficiency even at low pressures. Silicon nitride films have been deposited by $\mathrm{rf}$ reactive sputtering with ion assistance from an ECR plasma. The ECR power was varied from 0 to $250 \mathrm{~W}$. The effect of ECR plasma on the growth of silicon nitride films has been shown to be systematic and is characterized by using Fourier-transform infrared spectroscopy and atomic force microscopy. The surface roughness for the films deposited without ECR plasma was about $2 \mathrm{~nm}$ and decreased to about $0.7 \mathrm{~nm}$ for those deposited with $250 \mathrm{~W}$ of ECR power. The optical band gap changed from 2.2 to $4.9 \mathrm{eV}$ as the ECR power was increased, indicating higher reactivity in the presence of ECR plasma. Good quality silicon nitride films with a band gap of $4.9 \mathrm{eV}$, a refractive index of 1.92, and an extinction coefficient of $4 \times 10^{-4}$ have been obtained with an ECR power of $100 \mathrm{~W}$ (corresponding ion density of $1.5 \times 10^{10} \mathrm{~cm}^{-3}$ ) and the surface roughness was less than 0.7 $\mathrm{nm}$. The compositional analysis carried out using Auger electron spectroscopy showed a Si/N ratio of 0.73 for the films deposited under optimum deposition conditions.
\end{abstract}

\section{INTRODUCTION}

Silicon nitride $\left(\mathrm{Si}_{3} \mathrm{~N}_{4}\right)$ films are widely used in the fabrication of microelectronic devices due to their superior properties such as high thermal stability, chemical inertness, hardness, and good dielectric behavior. ${ }^{1-3}$ Presence of hydrogen in the silicon nitride films has been shown to be detrimental to the performance of the devices. Hydrogen will be present in the form of $\mathrm{Si}-\mathrm{H}$ and $\mathrm{N}-\mathrm{H}$ bonded groups and acts as deep traps or recombination centers. Conventionally silicon nitride films were deposited using a wide range of chemical vapor deposition (CVD) processes that use precursors such as silane, ammonia, etc. ${ }^{4-7}$ Even though CVD is widely used for the deposition of $\mathrm{Si}_{3} \mathrm{~N}_{4}$ films, the main disadvantages of this technique are the incorporation of hydrogen in the films and high substrate temperature. The entrapped hydrogen in the films can deteriorate the properties of $\mathrm{Si}_{3} \mathrm{~N}_{4}$ and the high substrate temperature is not generally desired in microelectronic applications. Reactive sputtering at low substrate temperature can provide $\mathrm{Si}_{3} \mathrm{~N}_{4}$ films with extremely low hydrogen content. 8,9 Added to that the properties of the films can be tailored with ion assistance. But the main problem encountered in ion-assisted deposition of $\mathrm{Si}_{3} \mathrm{~N}_{4}$ films is that the ion bombardment during the film growth induces lot of defects in the film which in turn degrade the electrical properties. ${ }^{10}$ These defects are due to the higher energy and flux of the ions bombarding the growing film. Thus a flexibility in controlling the flux and energy of the ions will facilitate in the growth of thin films of desired properties. In this respect electron cyclotron resonance (ECR) plasma, which is commonly used for plasma-

${ }^{a)}$ Electronic mail: gmrao@isu.iisc.ernet.in enhanced chemical vapor deposition (PECVD) provides an excellent choice. The low energy high density plasma, which is produced by an ECR ion source can be used for the ionassisted deposition of thin films. The high reactivity of the gas species and low pressure operation is an added advantage. To the best of our knowledge there are not many reports on the successful use of ion bombardment to deposit good quality $\mathrm{Si}_{3} \mathrm{~N}_{4}$ films. We have been able to deposit stoichiometric silicon nitride films using ECR plasma assisted sputter deposition. ${ }^{13}$ This article deals with the effect of ECR ion assistance on the structure and morphology of $\mathrm{Si}_{3} \mathrm{~N}_{4}$ films.

\section{EXPERIMENT}

In the present study we have used an ECR plasma assisted sputter deposition system developed in our laboratory. The details of the ECR system and the plasma diagnostics have been discussed in our earlier publication ${ }^{11}$ and Fig. 1 shows the salient features of the system. Microwaves from a magnetron source $(2 \mathrm{~kW})$ are transmitted into the source chamber through a quartz window. The magnetic mirror is provided by the two electromagnetic coils surrounding the source chamber. High-purity argon (99.999\%) and nitrogen (99.99\%) are introduced into the source chamber through mass flow controllers. The plasma generated in the source chamber diverges along the magnetic field lines, through an aperture of $100 \mathrm{~mm}$ diam. A magnetron sputter cathode is fixed with a $100 \mathrm{~mm}$ diam silicon target and powered with rf. Single-crystal Si (100) and polished silica substrates have been mounted at a distance of $100 \mathrm{~mm}$ orthagonally from the target on a heated platform kept at $350{ }^{\circ} \mathrm{C}$. This arrangement facilitates direct bombardment of ECR plasma on the grow- 


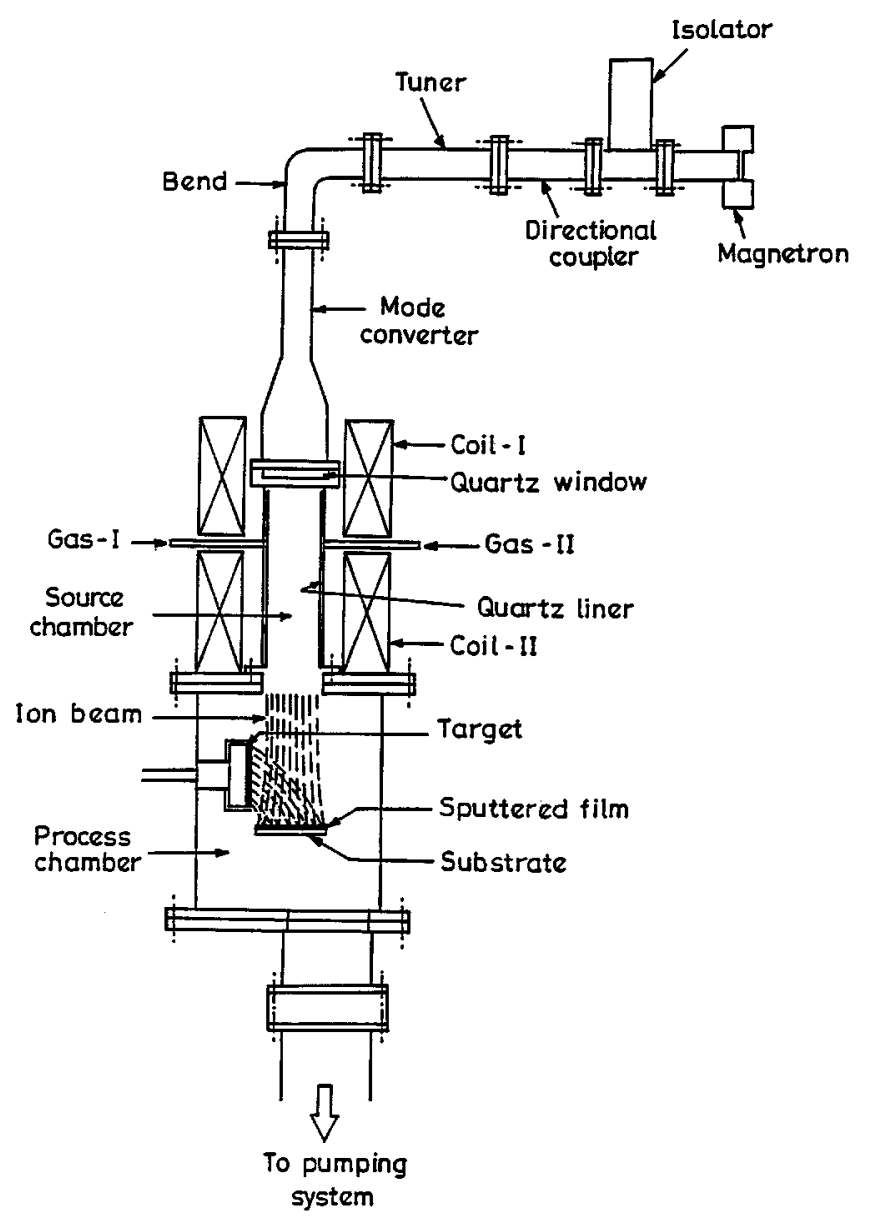

FIG. 1. Schematic of the ECR plasma assisted sputtering system.

ing film. Silicon nitride films $250 \mathrm{~nm}$ thick have been deposited and characterized using Fourier-transform infrared (FTIR) spectroscopy and atomic force microscopy (AFM). Compositional analysis has been carried out using Auger electron spectroscopy (AES) and Rutherford backscattering (RBS). The fundamental band gap is calculated using the transmittance data in the UV-VIS spectral region. All the films have been deposited with an rf power of $220 \mathrm{~W}$, nitrogen partial pressure of $2.5 \times 10^{-4} \mathrm{mbar}$, and a total pressure of $1.5 \times 10^{-3}$ mbar. Films have been prepared without ECR plasma and also with the ECR power varying from 50 to 250 $\mathrm{W}$, corresponding to an ion density on the substrate of 1.2 $\times 10^{10}$ to $2.4 \times 10^{10} \mathrm{~cm}^{-3}$. This corresponds to the low density ECR plasma regime. ${ }^{12}$

\section{RESULTS AND DISCUSSION}

\section{A. Compositional analysis}

The compositional analysis using AES and RBS agreed well within experimental error. Table I shows the results of compositional analysis of the films prepared at different ECR powers. The films prepared at different ECR powers are identified as sample 1, sample 2, etc. for convenience in further discussion. As seen from the table, the films prepared with zero ECR power are silicon rich. The amount of nitro-
TABLE I. Composition of silicon nitride films prepared at different ECR powers.

\begin{tabular}{ccc}
\hline \hline Sample No. & ECR power $(\mathrm{W})$ & Compostion $(\mathrm{Si} / \mathrm{N})$ \\
\hline 1 & 0 & 1.00 \\
2 & 50 & 0.82 \\
3 & 100 & 0.73 \\
4 & 200 & 0.68 \\
5 & 250 & 0.85 \\
\hline \hline
\end{tabular}

gen in the sample increases as the ECR power is increased. This increase in nitrogen content in the film is due to the higher reactivity of ECR plasma. At $100 \mathrm{~W}$ the ratio of $\mathrm{Si} / \mathrm{N}$ is about 0.73 as seen from the compositional analysis, which corresponds to nearly stoichiometric $\mathrm{Si}_{3} \mathrm{~N}_{4}$. Films deposited with higher ECR power also showed an increase in silicon content, which can be attributed to the resputtering of nitrogen due to the bombardment of higher ion flux. Though the films deposited without ECR assistance and with high-power ECR plasma are silicon rich, the nature of the chemical bonding in these films is different. X-ray photoemission spectroscopy (XPS) studies carried out on the samples showed this difference. Figure 2 shows the XPS spectra of samples 1,3 , and 5 corresponding to the Si $2 p$ core level. The Si $2 p$ core level for sample 1 is at $101.6 \mathrm{eV}$ and for samples 3 and 5 is at $102.2 \mathrm{eV}$, which corresponds to $\mathrm{Si}_{3} \mathrm{~N}_{4}$. The shift of $0.6 \mathrm{eV}$ indicates a lesser number of $\mathrm{Si}-\mathrm{N}$ bonds ${ }^{13}$ in sample 1 compared to samples 3 and 5. It means that the chemical environment of silicon in sample 1 differs from that in samples 3 and 5. The compositional analysis clearly shows that for a given set of deposition conditions such as sputtering pressure, target-substrate distance, and substrate temperature the film composition depends on the ion flux.

\section{B. Spectral studies}

The FTIR spectra (in the range of $400-4000 \mathrm{~cm}^{-1}$ ) of the silicon nitride films deposited at different ECR powers are shown in Fig. 3. All the spectra are corrected for substrate

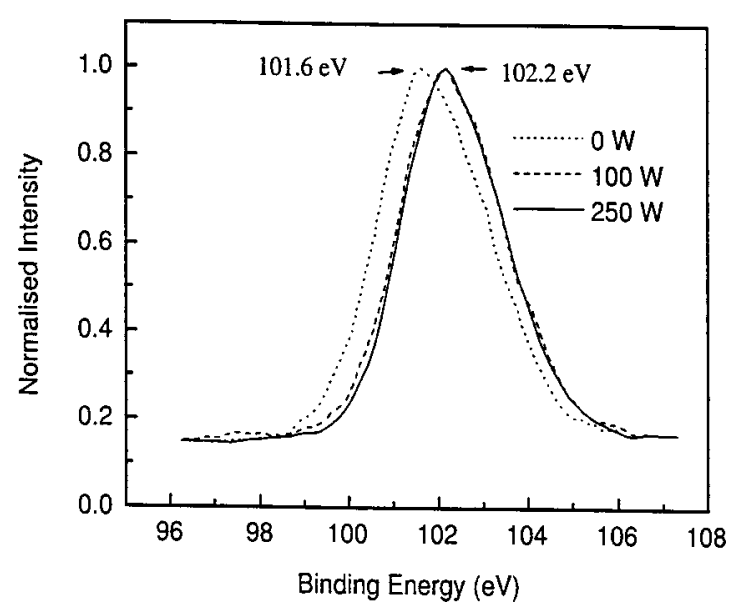

FIG. 2. XPS spectra of the sample prepared at 0, 100, and $250 \mathrm{~W}$. 


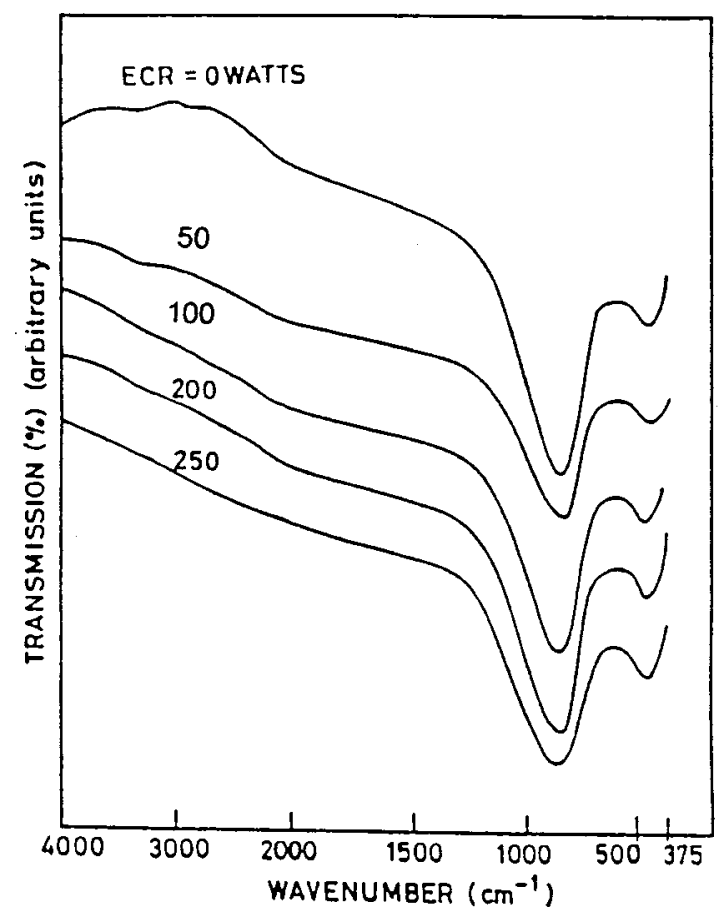

FIG. 3. FTIR spectra of silicon nitride films deposited at different ECR powers.

absorption. The spectra for the films deposited without ECR assistance and with low ECR power $(50 \mathrm{~W})$ show a small broad absorption band at $3340 \mathrm{~cm}^{-1}$. This band is associated with hydrogen bonding groups. The origin of this bonding was normally attributed to the precursors used in the process or due to water vapor absorption by the silicon nitride films after deposition. Generally films deposited with ion assistance would have more packing density and hence the water vapor absorption would be minimal. This is the reason for the absence of this band for the films deposited at higher ECR powers. Another feature of the spectra is the presence of a weak broad absorption band around $2190 \mathrm{~cm}^{-1}$. This is due to a $\mathrm{Si}-\mathrm{N}_{2}$ stretching band. ${ }^{14}$ All the films showed an absorption peak in the range of $847-865 \mathrm{~cm}^{-1}$. This band corresponds to the stretching vibration mode of the $\mathrm{Si}-\mathrm{N}$ bond. ${ }^{15}$ Another less prominent band is the one at $480 \mathrm{~cm}^{-1}$ which is due to the breathing mode of the $\mathrm{Si}-\mathrm{N}$ bond. ${ }^{16}$ However, one noticeable feature of these spectra is the consistent shift in the absorption peak to higher wave number with increasing ECR power, showing improved incorporation of nitrogen in the films.

The fundamental band gap of these films has been calculated from the optical spectra using Tauc's relation, ${ }^{17}$

$$
(\alpha h \nu)^{1 / 2}=A\left(h \nu-E_{g}\right),
$$

where $\alpha$ is the absorption coefficient, $A$ is a constant, $h \nu$ is the photon energy, and $E_{g}$ is the band gap. The extrapolation of the linear portion of the plot of $(\alpha h \nu)^{1 / 2}$ and photon energy $h \nu$ gives the value of the band gap. The band-gap values have been shown as a function of ECR power in Fig. 4. The films deposited without ECR plasma showed a lower band gap of $2.17 \mathrm{eV}$ which indicates nitrogen deficiency in

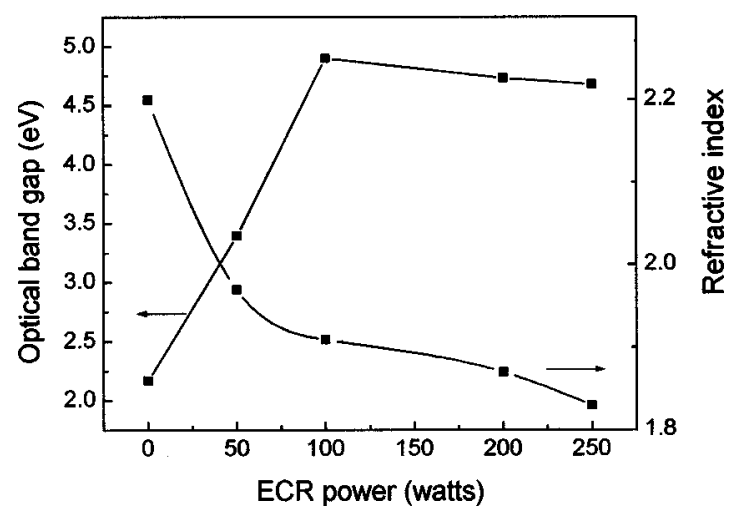

FIG. 4. Variation of fundamental band gap and refractive index in the silicon nitride films deposited at different ECR powers.

the films. With increase in the ECR power the nitrogen content in the films increases due to higher reactivity and is shown clearly by an increase in the band gap, which rises to about $4.9 \mathrm{eV}$ at a microwave power of $100 \mathrm{~W}$. This is in conformity with the band gap of bulk silicon nitride, which is about $5 \mathrm{eV}$. Further increase in the ECR power shows a slight reduction in the band gap and reaches a value of 4.7 $\mathrm{eV}$ for an ECR power of $250 \mathrm{~W}$. The optical transmittance of the films deposited with ECR assistance was good in the visible region reaching a maximum of 0.925 which is close to that of the fused silica substrate used. This is one of the important requirements of the silicon nitride films for mask applications in lithography. The spectral response showed a gradual shift with the increase in the incident ECR power. The decrease in the fundamental band gap and the shift in the infrared absorption peak beyond $100 \mathrm{~W}$ of ECR power could be due to an increase in short range disorder by displacement damage. Kim and Chung ${ }^{3}$ also observed similar trends during bias sputtering of silicon nitride films and they concluded that the enhanced ion bombardment leads to an increase in structural disorder in the silicon nitride films, thereby causing the optical band gap to decrease.

Using the envelope technique, the refractive index (at a wavelength of $650 \mathrm{~nm}$ ) of the films was calculated. ${ }^{18}$ The variation in the refractive index (Fig. 4) with microwave power is in accordance with the compositional analysis. The refractive index of the films deposited without ECR power is 2.2 and that for the sample prepared with $100 \mathrm{~W}$ ECR power is 1.92. The higher value of refractive index in the former case can be attributed to the excess amount of Si present in this sample (refractive index for bulk polished silicon is 4.05 and for silicon nitride is 2.02). For sample 3 the refractive index is 1.92 and is in agreement with the reported data. Kim and Chung ${ }^{3}$ have reported a refractive index between 1.9 and 2 for the silicon nitride films deposited by reactive bias sputtering.

\section{Surface morphology}

Ion bombardment on a growing film is known to induce morphological changes. To understand the surface topography of the films, AFM studies were carried out. Figures 

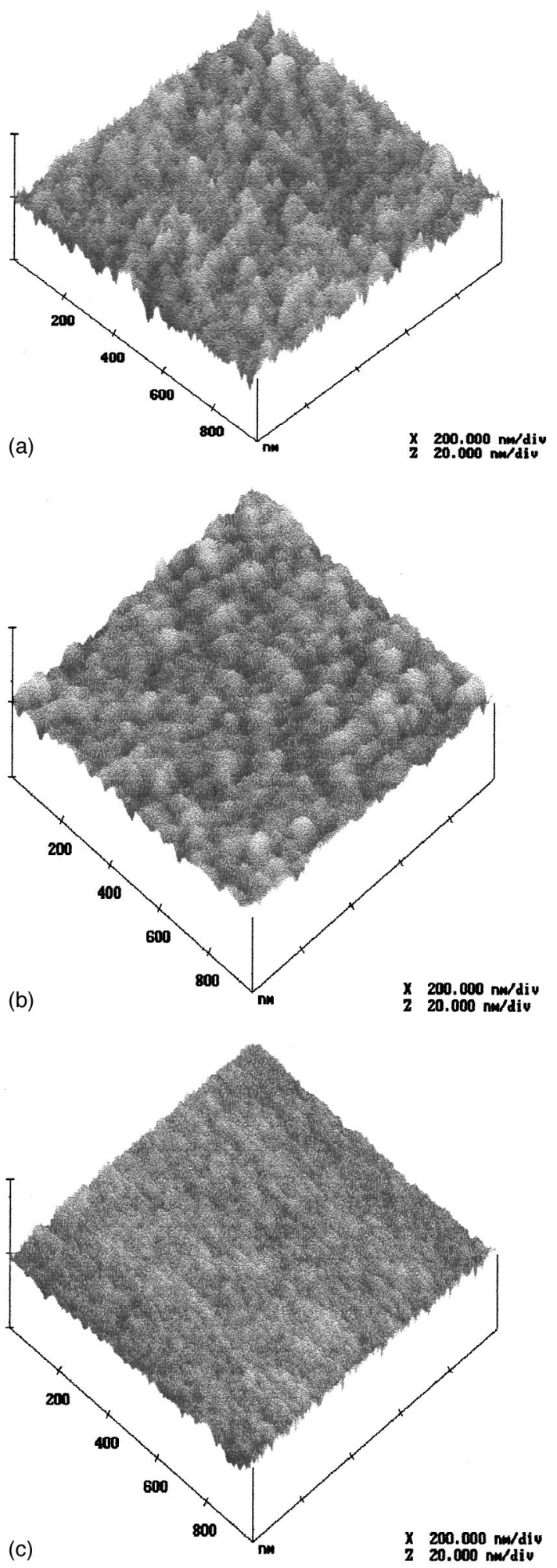

FIG. 5. Atomic force micrographs showing morphology of silicon nitride films deposited at different ECR powers; (a) without ECR, (b) $100 \mathrm{~W}$, and (c) $250 \mathrm{~W}$.

5(a)-5(c) show the three-dimensional AFM images of the films deposited without ECR plasma assistance and with 100 and $250 \mathrm{~W}$ of ECR power, respectively. The sampling area was $1 \times 1 \mu \mathrm{m}^{2}$ and about ten areas have been scanned on the surface to check the consistency of the images.

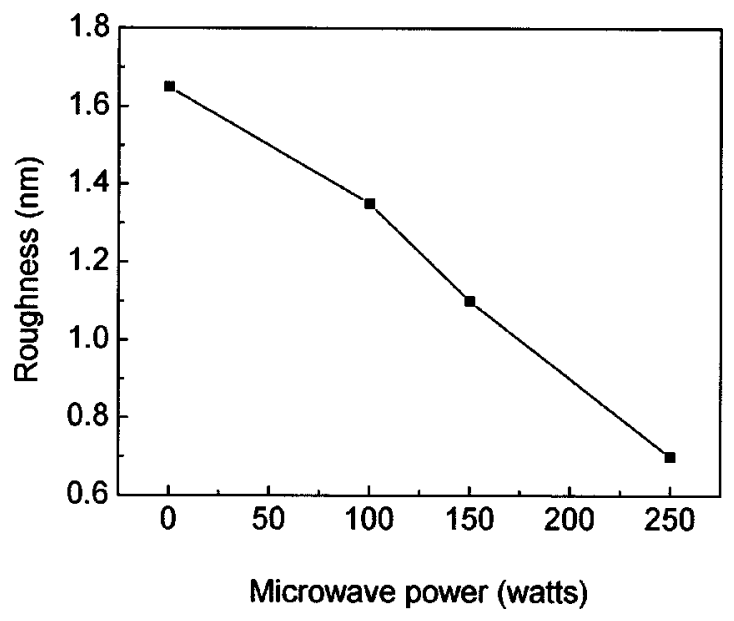

FIG. 6. Variation of the surface roughness with ECR power.

The films deposited without ECR assistance showed surface morphology with periodic projections exhibiting columnar growth of the films. According to the structure zone model this is characteristic of films grown at low pressures by sputtering. However, the films grown in the presence of ECR plasma show a remarkable reduction in these features and the films show a smooth morphology at an ECR power of $250 \mathrm{~W}$. This could be the zone T-like structure, devoid of any voids and inclusions due to enhanced mobility of the adatoms which can be attributed to ion bombardment from the ECR plasma. ${ }^{19,20}$ The roughness data of the films are shown in Fig. 6. The surface roughness for the films deposited without ECR plasma was about $2 \mathrm{~nm}$ and decreased to about $0.7 \mathrm{~nm}$ for those films deposited with $250 \mathrm{~W}$ of ECR power. This is consistent with the molecular dynamics models developed by Muller ${ }^{21}$ for ion-assisted growth of thin films. Kim and Chung ${ }^{3}$ reported similar behavior for silicon nitride films prepared by reactive bias magnetron sputtering. They observed that the surface roughness decreased drastically with increase in substrate bias. In general, resputtering, increased nucleation density, and increased adatom mobility under ion bombardment plays a major role in disrupting the columnar microstructure and this results in smooth surfaces. Similar results have been reported earlier in bias sputtered metal films. ${ }^{22}$

\section{CONCLUSIONS}

It was shown that the use of high-density and low-energy ECR plasma for ion-assisted growth in sputter deposition can be a useful tool in the growth of good-quality silicon nitride films with high optical transmittance and band gap. The variation of ion density from $1.2 \times 10^{10}$ to $2.4 \times 10^{10} \mathrm{~cm}^{-3}$ has been shown to have a significant effect on the growing films. Samples prepared at $100 \mathrm{~W}$ of ECR power were stoichiometric in nature, as confirmed by XPS and FTIR studies. Surface morphology studies showed that surface roughness decreases with ECR assistance. 


\section{ACKNOWLEDGMENTS}

The authors acknowledge the help of Dr. Ganesan (Inter University Consortium, Indore) for the atomic force microscopy studies. They are also thankful to AICTE, Government of India for financial assistance in developing the ECR source.

${ }^{1}$ T. P. Ma, IEEE Trans. Electron Devices 45, 680 (1998).

${ }^{2}$ N. Lustig and J. Kanicki, J. Appl. Phys. 65, 3951 (1989).

${ }^{3}$ A. K. Sinha, H. J. Levinstein, T. E. Smith, G. Quintana, and S. E. Haszko,

J. Electrochem. Soc. 125, 601 (1978)

${ }^{4}$ S. Trolier-McKinstry, H. Hu, and A. H. Carim, J. Electrochem. Soc. 141, 2483 (1994)

${ }^{5}$ S. V. Deshpande, E. Gulari, S. W. Brown, and S. C. Rand, J. Appl. Phys. 77, 6534 (1995)

${ }^{6}$ S. Sitbon, M. C. Hugon, B. Agius, F. Abel, J. L. Courant, and M. Puech, J. Vac. Sci. Technol. A 13, 2900 (1995).

${ }^{7}$ D. Landheer, K. Rajesh, D. Masson, J. E. Huise, G. I. Sproule, and T. Quance, J. Vac. Sci. Technol. A 6, 2931 (1998).
${ }^{8}$ S. K. Ray, S. Das, C. K. Maiti, S. K. Lahiri, and N. B. Chakraborti, J. Appl. Phys. 75, 8145 (1994).

${ }^{9}$ J. K. Kim and K. W. Chung, J. Appl. Phys. 83, 5831 (1998).

${ }^{10}$ M. F. Lambrinos, R. Valizadeh, and J. S. Colligon, J. Vac. Sci. Technol. B 16, 589 (1998).

${ }^{11}$ K. Deenamma Vargheese and G. Mohan Rao, Rev. Sci. Instrum. 71, 467 (2000).

${ }^{12}$ O. A. Popov, in Physics of Thin Films, edited by M. H. Francombe and J. L. Vossen (Academic, New York, 1994), Vol. 18.

${ }^{13}$ R. Karcher, L. Ley, and R. L. Johnson, Phys. Rev. B 30, 1896 (1984).

${ }^{14}$ L. Huang, K. W. Hipps, J. T. Dickison, U. Mazur, and X. D. Wang, Thin Solid Films 299, 104 (1997).

${ }^{15}$ G. N. Parsons, J. H. Souk, and J. Batey, J. Appl. Phys. 70, 1553 (1991).

${ }^{16}$ T. Serikawa and A. Okamoto, J. Electrochem. Soc. 131, 2928 (1984).

${ }^{17}$ J. Bauer, Phys. Status Solidi A 39, 411 (1977).

${ }^{18}$ R. Swanpoel, J. Phys. E 16, 1214 (1983).

${ }^{19}$ J. A. Thornton, Annu. Rev. Mater. Sci. 7, 239 (1977).

${ }^{20}$ R. F. Messier, A. P. Giri, and R. A. Roy, J. Vac. Sci. Technol. A 2, 500 (1984).

${ }^{21}$ K. H. Muller, J. Appl. Phys. 62, 1796 (1987).

${ }^{22}$ D. M. Mattox, J. Vac. Sci. Technol. A 7, 1105 (1989). 